\title{
Eficacia de extracto etanólico de eucalipto (Eucaliptus globulus) en el control de Alternaria sp. en cultivos de col y patata
}

\author{
María-Elena Cazar ${ }^{1}$, Paulina Villena ${ }^{2,3}$, Juan Parra ${ }^{3,4}$, Virgilio Espinoza ${ }^{3,4}$, Giovanni Larriva ${ }^{1,3}$, \\ Adriana Caldas ${ }^{3}$ \\ ${ }^{1}$ Facultad de Ciencias Químicas, Universidad de Cuenca, Av. 12 de Abril s/n, Cuenca, Ecuador. \\ ${ }^{2}$ Facultad de Ciencias Agropecuarias, Universidad de Cuenca, Campus Yanuncay. \\ ${ }^{3}$ Programa Control de Plagas en Cultivos Hortícolas de la Región Centro Sur del Ecuador, Dirección \\ de Investigación de la Universidad de Cuenca (DIUC), Av. 12 de Abril s/n, Cuenca, Ecuador. \\ ${ }^{4}$ Profesor Jubilado, Facultad de Ciencias Químicas, Universidad de Cuenca, Av. 12 de Abril s/n, \\ Cuenca, Ecuador.
}

Autor para correspondencia: maria.cazar@ucuenca.edu.ec

Fecha de recepción: 3 de julio de 2013 - Fecha de aceptación: 6 de marzo 2014

\begin{abstract}
RESUMEN
El efecto inhibitorio de extractos etanólicos ante Alternaria sp. en cultivos de invernadero de col (Oleracea brassica) y crecimiento in-situ de patatas (Solanum tuberosum) fue evaluado. Se prepararon extractos a partir de hojas de eucalipto (Eucalyptus globulus) por el método Soxhlet. El análisis fitoquímico de los extractos reveló la presencia de cantidades considerables de quinonas, lactonas y cumarinas y bajas cantidades de triterpenos y esteroides. El diseño experimental en el cultivo de invernadero de col consistió de tres niveles de concentración de extracto etanólico, un tratamiento químico (Trizimand) y un control; 10 coles por tratamiento y tres repeticiones, dispuestas en un diseño de bloques completos al azar. El efecto de tres niveles de concentración de extracto etanólico y un tiempo de aplicación en las hojas de plantas de patata fue probado. Los grupos de tratamiento, incluyendo un control, fueron aleatorizados con cuatro repeticiones cada uno. En el ensayo de invernadero, las dosis efectivas incrementadas en un 50 y $25 \%$ mostraron una actividad comparable al tratamiento químico, y fue superior en efectividad a los tratamientos con dosis efectiva en condiciones de laboratorio. En el ensayo de campo en patata, basado en la incidencia de pústulas medido en un período de 65 a 85 días después de la siembra, todos los tratamientos mostraron igual efecto. Es posible que el menor efecto observado en los extractos etanólicos aplicados en el cultivo de patata se deba a la elevada variabilidad entre bloques y el nivel de precipitaciones mayor al normal durante la estación de crecimiento del cultivo.
\end{abstract}

Palabras clave: Eucalyptus globulus, control biológico, género Alternaria, ensayo de campo, ensayo de invernadero.

\begin{abstract}
The inhibitory effect of ethanolic extracts against Alternaria sp. in greenhouse cabbage cultivation (Oleracea brassica) and in-situ potato growing (Solanum tuberosum) was tested. Ethanolic extracts were prepared from Eucalyptus leaves (Eucalyptus globulus) with a Soxhlet extractor. Phytochemical analysis revealed the presence of considerable amounts of quinones, lactones and coumaranes, and small amounts of triterpenes and steroids. The experimental layout in the greenhouse cabbage cultivation consisted of three ethanol levels, one classical chemical treatment (Trizimand) and one control, ten cabbages per treatment, three repetitions, arranged in a randomized complete block design. The effect of three concentration levels of ethanol, one time sprayed on the leaves of the potato crop, was tested. The treatment plots, including a control plot, were randomized, each block 4 times repeated. In the greenhouse trial, the treatments with the effective dose increased by 50 and $25 \%$
\end{abstract}


displayed a comparable activity with the chemical treatment. Those treatments showed an increased effectiveness, compared with the effective dose at lab conditions. In the potato field essay, based on the incidence of pustules measured in the period of 65 to 85 days after planting, all treatments had an equal effect. It is believed that the observed overall minor and equal impact of the ethanol treatments on the potato crop is due to the high plot-to-plot variability and the more than normal wet conditions during the growing season.

Keywords: Eucalyptus globulus, biologic control, genus Alternaria, greenhouse experiment, field experiment.

\section{INTRODUCCIÓN}

Las enfermedades de plantas, causadas por hongos, bacterias, virus, protozoos, nematodos y plantas parásitas son uno de los mayores problemas relacionados al bajo rendimiento de cultivos. Por estas razones, las estrategias dirigidas a combatir y mitigar el efecto causado por el ataque de fitopatógenos es una importante consideración en la producción de especies de importancia económica (Kotan y col., 2013). Los fungicidas son considerados componentes fundamentales para el control efectivo de enfermedades de plantas causadas por hongos cuales, se estima, pueden reducir el rendimiento de producción de cultivos agrícolas hasta en un 20\%. Desde su introducción en los años 60, los fungicidas sistémicos han reemplazado gradualmente a los antiguos productos no sistémicos, estableciendo altos niveles de control de enfermedades y desarrollando nuevos mercados de fungicidas. Durante los últimos años, la investigación en fungicidas ha producido un amplio rango de productos con nuevos mecanismos de acción, los cuales se espera tengan un impacto significativo en el control de plagas. Estos nuevos productos incluyen anilinopirimidinas, fenoxiquinolinas, oxazolidinedinonas, espiroquetalaminas, estrobilurinas y activadores de resistencia sistémica adquirida (SAR) (Gullino y col., 2000).

La búsqueda de estrategias para incrementar el rendimiento de cultivos de importancia económica ha sido asociada a la "revolución verde", época en la cual el descubrimiento de productos químicos para control de enfermedades vegetales fue ampliamente aceptado y aplicado en el agro. Actualmente, las preocupaciones generadas por los efectos observados en el ambiente y la salud humana, derivados por el uso indiscriminado de pesticidas de síntesis química, han enfocado el interés de la sociedad y la comunidad científica en el desarrollo de nuevas alternativas al control de fitopatógenos. El paradigma de confiar casi exclusivamente en productos de síntesis química para el combate a las plagas agrícolas es paulatinamente reemplazado por el uso de productos basados en extractos vegetales, microorganismos antagonistas y técnicas de control integrado de plagas (Dayan y col., 2009). El interés en el control biológico de patógenos vegetales se ha incrementado considerablemente en los últimos años, parcialmente como una respuesta a la preocupación pública en relación al uso de pesticidas químicos peligrosos, pero también debido a los beneficios que provee el control biológico, en relación a otras estrategias de control. El manejo de microorganismos antagonistas, la rotación de cultivos y mejoras orgánicas han sido señaladas como formas altamente efectivas de control biológico en cultivos (Raaijmakers y col., 2002).

Entre las alternativas exploradas para el control biológico, los productos naturales son considerados ambientalmente seguros para el control de enfermedades causadas por fitopatógenos. Las plantas superiores son un repositorio de fitoquímicos que sirven como principios activos valiosos que han ayudado a combatir enfermedades a nivel mundial. Las plantas superiores incluyen entre sus mecanismos de defensa la fitotoxicidad, como estrategia de control ante la germinación de esporas y el crecimiento micelial de hongos fitopatógenos. Algunos de los metabolitos de plantas superiores han sido reportados como altamente efectivos como inhibidores del crecimiento de patógenos vegetales (Satish y col., 2009; Khajista y Javaid, 2008). En Ecuador se registra una demanda creciente de plaguicidas para el control de enfermedades de plantas. Según la FAO el valor de las importaciones de plaguicidas se multiplicó 47 veces en el período de 1972 a 2002 (Gaybor y col., 2006). En este proceso se generan daños ambientales y a poblaciones no objetivo, los cuales se asocian a problemas de salud 
de los agricultores y tienen consecuencias no cuantificables en el ecosistema megadiverso ecuatoriano (Quintana y col., 2008).

Varios biopesticidas eco-compatibles son formulados a partir de extractos vegetales, los cuales son mezclas de principios activos de plantas obtenidos mediante extracción con un solvente apropiado, el cual es evaporado y el residuo debe ser ajustado a una concentración previamente establecida en ensayos in vitro (Koul y Walia, 2009). En este contexto, las investigaciones orientadas a probar la efectividad de extractos vegetales como agentes biocontroladores de fitopatógenos en condiciones de invernadero y campo constituye una aproximación válida para recomendar el uso de estos bioproductos en el control de plagas comunes en cultivos de importancia económica.

Eucalyptus (familia Myrtaceae) es un género que agrupa a especies forestales nativas de Australia, representado por alrededor de 700 especies. Este género de árboles altos, siempre verdes, son cultivados en todo el mundo por el valor de su madera y su potencial en el desarrollo de medicamentos y perfumes. Los compuestos biosintetizados por los miembros de esta especie ejercen un efecto alelopático en el ecosistema vegetal, inhibiendo el crecimiento de especies competidoras, debido a la producción de varios aleloquímicos: p-mentano-3,8-dioles, eucaliptina, $\beta$-sitosterol y triterpenoides (Jabeen y Javaid, 2008). Los extractos acuosos, hexánicos y metanólicos de Eucalyptus citrodiora inhiben el crecimiento micelial de hongos fitopatógenos que afectan cultivos de arroz, como Bipolaris oryzae y Didymella byroneae (Harish y col., 2008). Además, numerosos trabajos científicos presentan evidencias en relación al efecto antifúngico de extractos vegetales y aceites esenciales de especies del género Eucalyptus. El aceite esencial de eucalipto posee un amplio espectro de actividades biológicas, incluyendo antimicrobiana, antifúngica, insecticida, repelente, herbicida, acaricida y nematicida (Batish y col., 2008). Trabajos previos de nuestro grupo de investigación reportaron la dosis mínima inhibitoria de extractos orgánicos de E. globulus en ensayos in vitro (Cazar y Parra, 2012).

Los resultados promisorios obtenidos en los ensayos en condiciones de laboratorio motivaron el desarrollo de pruebas de invernadero y campo. El objetivo planteado fue establecer la dosis efectiva a aplicarse en cultivos, donde los factores ambientales pueden afectar la estabilidad y modificar la eficiencia de los biopesticidas, en comparación a las condiciones in vitro. El patógeno objetivo pertenece al género Alternaria, conformado por hongos cosmopolitas, que causan diferentes enfermedades en sus huéspedes. A. solani causa el tizón temprano de la papa (Solanum tuberosum) y tomate (Licopersicum esculentum). Las infecciones con este patógeno causan daños en el follaje, damping-off, lesiones en los tallos y frutos de varias especies. Estas enfermedades pueden ser muy destructivas, causando a menudo la completa defoliación de las plantas (Aslam y col., 2010).

En este trabajo se evalúa la efectividad del extracto etanólico de eucalipto como biocontrolador de Alternaria sp., en condiciones de invernadero y campo; en hortalizas de cultivo masivo en la sierra sur ecuatoriana. A continuación se revisará la metodología empleada y los resultados obtenidos que permiten señalar la factibilidad del uso de extractos de eucalipto en el control de Alternaria sp. en cultivos hortícolas.

\section{MATERIALES Y MÉTODOS}

\subsection{MATERIALES}

Descripción del área experimental

La presente investigación se llevó a cabo en áreas urbanas y rurales de Cuenca, Ecuador. Las muestras de hortalizas contaminadas fueron obtenidos de cultivos hortícolas de la Parroquia San Joaquín. Los extractos vegetales fueron preparados en el Laboratorio de Biotecnología, Campus Balzaín, Universidad de Cuenca. En este campus se acondicionaron áreas experimentales para el ensayo de invernadero y campo. En el invernadero se desarrollaron cultivos de col (Brassica olearacea Alba) y en campo se utilizó como organismo de prueba plantas de papa (Solanum tuberosum), variedad local "chaucha", proveniente de semillas certificadas. 


\subsection{MÉTODOS}

\section{Aislamiento y caracterización del microorganismo de prueba}

El hongo fitopatógeno Alternaria sp. fue aislado de hojas de col (Brassica oleracea) con síntomas visibles de la enfermedad. Las pústulas fueron cortadas y transferidas asépticamente, previa desinfección en etanol $(70 \% \mathrm{v} / \mathrm{v})$, en placas petri con Agar Papa-Dextrosa (agar base 2\%, papa deshidratada $1,5 \%$, dextrosa $1,5 \%, \mathrm{pH}=5,5$ ), con adición de nistatina y cloranfenicol, con el fin de evitar el crecimiento de bacterias y hongos ambientales. Las placas petri sembradas fueron incubadas a $25^{\circ} \mathrm{C}$ por 8 días. Luego de varios repiques sucesivos se seleccionaron aislados puros, los cuales fueron analizados para identificar el patógeno en sus características macro y microscópicas. Macroscópicamente, se observaron la apariencia y colocación característica del género Alternaria. Para las características microscópicas se realizaron observaciones de micelio e hifas en microscopio, usando un aumento de 40x. Las características del micelio fueron observadas extrayendo una muestra con cinta adhesiva y utilizando como colorante de contraste azul de lactofenol. Adicionalmente, se realizó un microcultivo utilizando la técnica de cámara de humedad. Mediante esta estrategia se observaron estructuras fungales (conidios, conidióforos e hifas), las cuales fueron contrastadas con claves de identificación y se confirmó el género de los aislados del fitopatógeno de prueba.

\section{Obtención y caracterización del extracto etanólico de Eucaliptus globulus}

Se recolectaron partes aéreas de E. globulus, en un bosque ubicado en la Parroquia Ricaurte, Cuenca. Las hojas fueron secadas al ambiente, protegidas de exposición directa a la luz solar y la humedad. Posteriormente, se separaron las nervaduras y se pulverizó el material vegetal en un molino manual. La extracción exhaustiva del material vegetal se realizó utilizando un solvente de elevada polaridad, etanol, mediante un proceso continuo en un extractor Soxhlet (Soxhlet, 1879).

El extracto etanólico fue caracterizado cualitativamente mediante pruebas fitoquímicas. Se desarrollaron ensayos de identificación de azúcares reductores mediante la prueba de Fehling (Fehling, 1849); la prueba de generación de espuma en una solución acuosa del extracto, atribuida a la presencia de saponinas. Se ensayó la presencia de alcaloides mediante el ensayo de Dragendorff (Daintith, 2008) y se evaluó la presencia de taninos, aminoácidos y flavonoides mediante las pruebas de cloruro férrico, ninhidrina y Shinoda (Mallikharjuna y col., 2007), respectivamente. Adicionalmente, la presencia de quinonas, esteroides y antocianidinas fue evaluada con ensayos fitoquímicos preliminares: pruebas de Baljet, Borntrager, Lieberman-Burchard y Rosenheim (Corral Salvado y col., 1997), respectivamente.

\section{Ensayo de invernadero}

Con el fin de evaluar la eficiencia del extracto etanólico de $E$. globulus y ajustar la dosis efectiva para el ensayo de campo, se realizó un ensayo de invernadero. Se prepararon macetas con plántulas de col usando sustrato preparado a partir de tierra negra estéril y turba (proporción 2:1). Las macetas fueron regadas hasta capacidad de campo y se monitorearon antes del desarrollo del ensayo, para verificar la viabilidad de las plántulas antes del ensayo. En el invernadero las condiciones de temperatura y humedad fueron $25^{\circ} \mathrm{C} \pm 2^{\circ} \mathrm{C}$ y $70 \%$ de humedad relativa.

Veintisiete días posteriores a la siembra y adaptación de plántulas en macetas, una solución de esporas del hongo patógeno $\left(2 \times 10^{5}\right.$ esporas $\left.\mathrm{ml}^{-1}\right)$ fue inoculado mediante aspersión en atomizadores. En este ensayo se utilizó un diseño estadístico de bloques al azar (DBA) con tres repeticiones. El ensayo de invernadero en col (B. oleracea) consistió en la prueba de tres niveles de concentración de extracto etanólico, cuya dosis efectiva fue establecida previamente en $200 \mathrm{mg} \mathrm{l}^{-1}$, tomando como referencia el trabajo de Cazar y Parra (2012). Se establecieron tratamientos con diferentes concentraciones de extracto (Tratamiento A: dosis efectiva incrementada en un 50\%: $300 \mathrm{mg} \mathrm{l}^{-1}$; Tratamiento B: dosis efectiva incrementada en un $25 \%, 250 \mathrm{mg} \mathrm{l}^{-1}$; Tratamiento C: dosis efectiva de

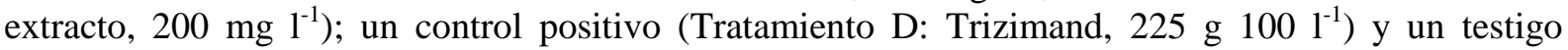
(Tratamiento E). Cada tratamiento fue asignado aleatoriamente en un bloque, constituido por un número variable de macetas. En los tratamientos de control positivo y testigo se asignaron 10 macetas por bloque y en los tratamientos de las dosis de extracto (A, B y C), 30 macetas por bloque. Los bloques fueron repetidos por triplicado, constituyendo un experimento de bloques completos al azar. 
El extracto alcohólico de eucalipto fue diluido a las concentraciones establecidas en el ensayo con agua, adicionando como dispersante sodio dodecil sulfato a una concentración de $2 \% \mathrm{v} / \mathrm{v}$. Las aspersiones se realizaron directamente en el follaje de las plantas con la ayuda de un atomizador.

\section{Ensayo de campo}

Para el ensayo de campo, una extensión de $260 \mathrm{~m}^{2}$, ubicada a 2550 m.s.n.m fue dividida en cuatro bloques de 2,70 m de ancho por $19 \mathrm{~m}$ de largo; cada bloque se subdividió en cuatro parcelas de 10,8 $\mathrm{m}^{2}$. El terreno seleccionado para el ensayo no registra desarrollo de cultivos previos y no se verifican cultivos similares en los alrededores. El suelo presenta una capa arable superior de $50 \mathrm{~cm}$. Previo a la siembra se preparó el suelo mediante arado con yunta, incorporando residuos vegetales y controlando malezas. La siembra se realizó 15 días después de estas labores, permitiendo una adecuada descomposición de los residuos vegetales incorporados. Se utilizó semilla vegetativa de patata variedad "chaucha", empleando alrededor de $12 \mathrm{~kg}$ de semilla para un área de $260 \mathrm{~m}^{2}$. Se realizó la siembra a una distancia de $40 \mathrm{~cm}$ entre plantas y 50 entre surcos. La temperatura media y humedad ambiental fueron de $16^{\circ} \mathrm{C}$ y $64 \%$, respectivamente. En el período de cultivo (Diciembre 2011 - Marzo 2012), se registraron récords de precipitación acumulada mensual, superiores a los de un año normal (INHAMI, 2012).

Cincuenta días posteriores a la siembra se realizó el aporque y la inoculación del patógeno de prueba por atomización, según lo descrito en el ensayo de invernadero. El experimento se desarrolló mediante un diseño de bloques completos al azar, en los cuales se asignaron aleatoriamente cuatro tratamientos, uno correspondiente al testigo y tres tratamientos con dosis de extracto en un rango de 220 a $420 \mathrm{mg} \mathrm{l}^{-1}$. El rango de concentraciones seleccionado oscila en torno a la dosis efectiva en invernadero, y se orienta a establecer la efectividad de estas dosis en la parcela de prueba. La respuesta experimental fue el número de pústulas por foliolos en todas las plantas, sin considerar el efecto borde de cada parcela experimental.

\section{RESULTADOS Y DISCUSIÓN}

\subsection{Caracterización del extracto etanólico de Eucalyptus globulus}

Las pruebas fitoquímicas cualitativas del extracto etanólico de E. globulus revelaron la presencia de quinonas, lactonas y coumarinas, como compuestos mayoritarios y menor cantidad de triterpenos y esteroides. La presencia de polifenoles, caracterizados en estudios previos, ha sido asociada a la actividad antioxidante de extractos de E. globulus. La actividad antioxidante está relacionada con el efecto antifúngico y antimicrobiano en los extractos en estudio (Amakura y col., 2009).

\subsection{Control de Alternaria sp. en cultivos de Brassica oleracea en condiciones de invernadero}

Los datos obtenidos en la prueba de invernadero, realizada según lo descrito en la sección 2.2; fueron analizados mediante Análisis de Varianza a una vía (Fischer, 1925). Las posibles diferencias entre tratamientos se analizaron con la prueba de intervalos múltiples de Duncan (Duncan, 1955). Se estableció la efectividad de los tratamientos en función al número de lesiones foliares debidas al ataque de Alternaria sp. en comparación con el testigo. A continuación se presentan los resultados obtenidos en un diagrama de caja (Fig. 1), evidenciando las diferencias de medias entre los tratamientos.

La hipótesis que establece no existencia de diferencias de promedios de efectividad entre tratamientos fue rechazada $(\mathrm{F}=14,39 ; \mathrm{p}<0,05)$. La prueba post-hoc de Duncan establece que los tratamientos A, B y D difieren significativamente del tratamiento $\mathrm{C}$ y el testigo. Por consiguiente, se puede inferir que el número de lesiones foliares de las plantas tratadas con los tratamiento $\mathrm{A}$ y $\mathrm{B}$ (concentración de extracto de E. globulus 300 y $250 \mathrm{mg} \mathrm{l}^{-1}$, respectivamente) es similar a la del control químico aplicado en este ensayo. Esta dosis es utilizada como base para la prueba de campo, cuyo objetivo es explorar un rango de concentraciones cercanas con el fin de establecer la eficiencia del 
extracto etanólico de E. globulus en condiciones ambientales, afectadas por factores bióticos y abióticos propios del ecosistema agrícola.

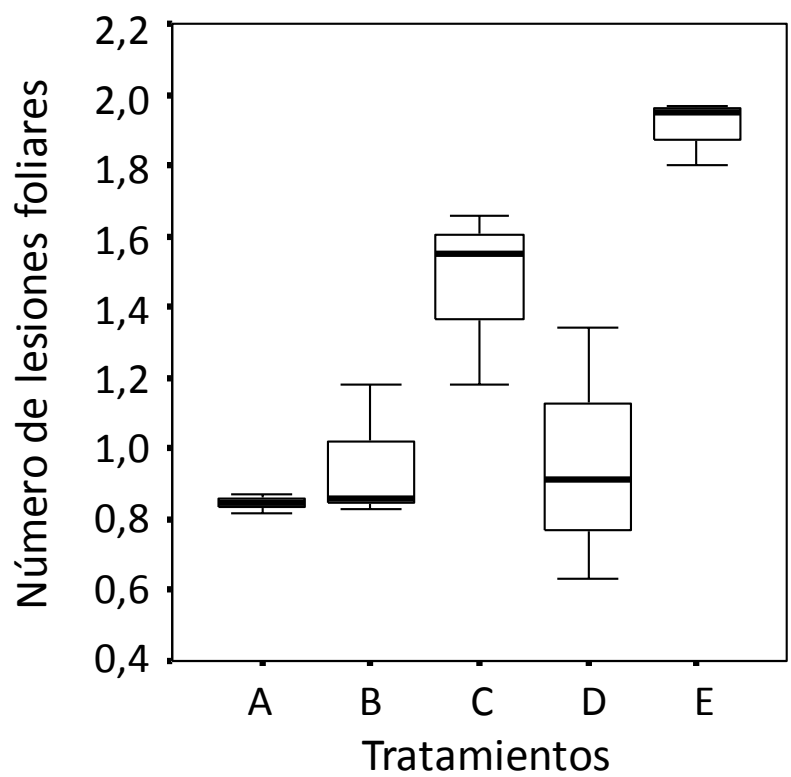

Figura 1. Comparación del número de lesiones foliares en col, de los tratamientos y controles en ensayo de invernadero. A: dosis efectiva $+50 \%$; B: dosis efectiva $+25 \%$; C: dosis efectiva; D: control químico; E: testigo.

\subsection{Control de Alternaria sp. en cultivos de Solanum tuberosum en campo}

La efectividad del extracto etanólico de E. globulus en el control del patógeno de prueba fue evaluada en parcelas experimentales, según lo descrito en la sección 2.2. A continuación se presenta un diagrama de caja (Fig. 2) que compara los niveles de infestación en pústulas foliares de los tratamientos y el testigo.

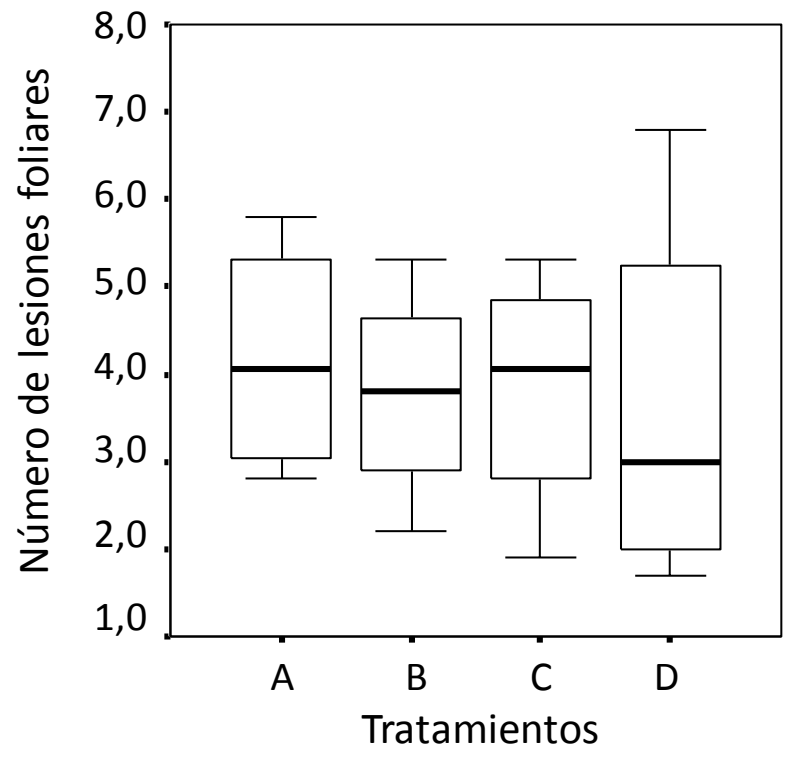

Figura 2. Comparación entre número de lesiones foliares de los tratamientos y el testigo en ensayo de campo. Concentraciones de extracto en los tratamientos: $\mathrm{A}=220 \mathrm{mg}^{-1} ; \mathrm{B}=320 \mathrm{mg}^{-1} ; \mathrm{C}=420 \mathrm{mg}$ $1^{-1}$; D: testigo. 
El gráfico revela que el número de lesiones foliares observado en las plantas con los tratamientos experimentales es similar. Esta observación es corroborada mediante los resultados obtenidos en el ANOVA $(\mathrm{F}=0,08 ; \mathrm{p}>0,05)$. El análisis estadístico muestra una considerable variabilidad entre los tratamientos. Esto es atribuible a las condiciones ambientales propias de la serranía ecuatoriana. Las abundantes precipitaciones pueden actuar como un dispersante del agente biocontrolador de prueba. Una de las características deseables en todo bioproducto agrícola es su estabilidad ante las condiciones de luminosidad propias de nuestra región. Varios metabolitos secundarios de un extracto vegetal tienden a degradarse ante la luz solar, disminuyendo la eficiencia observada en condiciones in vitro o de invernadero (Spadaro y Gullino, 2005).

Los resultados de la efectividad de extractos orgánicos vegetales como biocontroladores en condiciones in vitro pueden, en ocasiones, no ser replicables a condiciones in vivo. No obstante, la selección de biocontroladores mediante ensayos de laboratorio permite aproximarse a candidatos para el desarrollo de nuevos agentes de biocontrol y establecer posibles mecanismos de acción asociados al efecto observado en condiciones in vitro (Mejía y col., 2008). Esta información fundamenta el desarrollo de pruebas de campo e invernadero, cuyo desarrollo representa un desafío para la investigación agraria actual, ya que se trata de diseñar ensayos robustos que permitan ofrecer resultados que respalden el uso de productos naturales en el control de plagas y enfermedades de cultivos.

\section{CONCLUSIONES}

En la presente investigación se describe la metodología para el aislamiento de Alternaria sp. y su uso como patógeno de prueba en ensayos in vivo. El desarrollo del ensayo de invernadero permitió establecer una dosis activa de extracto de E. globulus, la cual fue testada en una prueba de campo, orientada al control de Alternaria sp. en cultivos de papa. Los resultados de invernadero fueron promisorios, ya que la dosis efectiva en laboratorio incrementada en un 50 y $25 \%$ (300 y $250 \mathrm{mg}^{-1}$ ) tuvo una eficiencia comparable al control químico. En la prueba de campo no se encontraron diferencias entre tratamientos, evidenciando que las condiciones experimentales incrementan la variabilidad de los resultados obtenidos y afectan la potencia de las pruebas estadísticas. Es necesario replicar estas experiencias a pesar de las dificultades que plantea el desarrollo de una prueba de campo. Sin el desarrollo de estas aproximaciones el uso de bioproductos en el agro no podría ser establecido como una práctica efectiva, considerando los efectos beneficiosos en el ambiente, la menor toxicidad para las poblaciones no-objetivo y el incremento de la seguridad de los alimentos cultivados prescindiendo de los controles químicos tradicionales.

\section{AGRADECIMIENTOS}

La presente investigación se realizó con el apoyo de la Agencia Cuencana para el Desarrollo Regional (ACUDIR) y el Proyecto P/00040946: "Proyecto para mejorar el Desarrollo Humano sostenible del Cantón Cuenca y la Provincia del Azuay a través de la reactivación productiva a fin de mejorar el mercado laboral" de las Naciones Unidas para el Desarrollo (PNUD), como entidades financiadoras de esta investigación.

\section{BIBLIOGRAFÍA}

Amakura, Y., M. Yoshimura, N. Sugimoto, T. Yamazaki, T. Yoshida, 2009. Marker constituents of the natural antioxidant Eucalyptus leaf extract for the evaluation of food additives. Biosci., Biotechnol.,Biochem., 73(5), 1060-1065. 
Aslam, A., M. Farah-Naz, R. Arshad, C. Rauf, 2010. In vitro antifungal activity of selected medicinal plant diffusates against Alternaria solani, Rhizoctonia solani and Macrophomina phaseolina. Pakistan J. Bot., 42(4), 2911-2919.

Batish, D., P. Harminder, K. Ravinder, K. Shalinder, 2008. Eucalyptus essential oil as a natural pesticide. For. Ecol. Manage., 256(12), 2166-2174.

Cazar, M., J. Parra, 2012. Extractos vegetales promisorios como biocontroladores de Botrytis cinerea y Alternaria sp. Biotecnología, 4, 29-32.

Corral Salvado, A., J. De la Paz Naranjo, E. Concepción Evseeva, R. Hernández Royero, D.L. López Rodríguez, 1997. Tamizaje, tecnología, control de calidad y farmacología del extracto fluido de Bouganvillea spetabilis Willd. Rev. Cubana Plant. Med., 2(2), 19-25.

Daintith, J. (Ed.), 2008. Dragendorff test. A dictionary of chemistry (6 ed.). Oxford University Press, $\mathrm{UK}, 592 \mathrm{pp}$.

Dayan, F., C. Cantrell, S. Duke, 2009. Natural products in crop protection. Bioorg. Med. Chem., 17(12), 4022-4034.

Duncan, D.B., 1955. Multiple range and multiple F tests. Biometrics, 11, 1-42.

Fehling, H., 1849. Die quantitative Bestimmung von Zucker und Stärkmehl mittelst Kupfervitriol. Ann. Chemie und Pharmacie, 72(1), 106-113.

Fisher, R.A., 1925. Statistical methods for research workers. Oliver \& Boyd, Edinburgh, UK, 362 pp.

Gaybor A., C. Nieto, R. Velasteguí, 2006. TLC y plaguicidas: Impactos en los mercados y la agricultura ecuatoriana. Sistema de Investigación sobre la Problemática Agraria en el Ecuador (SIPAE), Quito, Ecuador, 130 pp.

Gullino, M., P. Leroux, C. Smith, 2000. Uses and challenges of novel compounds for plant disease control. Crop Prot., 19(1), 1-11.

Harish, S., D. Saravanakumar, R. Radjacommare, E.G. Ebenezar, K. Seetharaman, 2008. Use of plant extracts and biocontrol agents for the management of brown spot disease in rice. BioControl, 53(3), 555-567.

INHAMI (2012). Boletín climatológico anual. Año 2012. Descargado de http://www.serviciometeorologico.gob.ec/ en febrero de 2014.

Jabeen, K., A. Javaid, 2008. Antifungal activity of aqueous and organic extracts of allelopatic trees against Ascochyta rabiei. Allelopath. J., 22(1), 231-237.

Khajista, J., A. Javaid, 2008. Antifungal activity of aqueous and organic solvent extracts of allelopathic trees against Ascochyta rabiei. Allelopath. J., 22, 231-238.

Kotan, R., F. Dadasoglu, K. Karagoz, A. Cakir, H. Ozer, S. Kordali, R. Cakmakci, N. Dikbas, 2013. Antibacterial activity of the essential oil and extracts of Satureja hortensis against plant pathogenic bacteria and their potential use as seed disinfectants. Sci. Hortic., 153, $34-41$.

Koul, O., S. Walia, 2009. Comparing impacts of plant extracts and pure allelochemicals and implications for pest control. CAB Reviews: Perspectives in Agriculture, Veterinary Science, Nutrition and Natural Resources, 4(049), 1-30.

Mallikharjuna, P.B., L.N. Rajanna, Y.N. Seetharam, G.K. Sharanabasappa, 2007. Phytochemical studies of Strychnos potatorum L.F., a medicinal plant. Electron. J. Chem., 4(4), 510-518.

Mejía, L., E. Rojas, Z. Maynard, S. Van Bael, E. Arnold, P. Hebbar, G. Samuels, N. Robbins, A. Edward, 2008. Endophytic fungi as biocontrol agents of Theobroma cacao pathogens. BioControl, 46(1), 4-14.

Quintana, G.Y., A. Noboa, D. Alava, T. Yánez-N., 2008. Manual técnico para la gestión integral de plaguicidas. Programa de apoyo a la gestión descentralizada de los recursos naturales en las tres provincias del norte del Ecuador: Carchi, Esmeraldas e Imbabura (PRODERENA). Ministerio del Ambiente, Quito, Ecuador, 160 pp.

Raaijmakers, J.M., M. Vlami, J.T. De Souza, 2002. Antibiotic production by bacterial biocontrol agents. Antonie van Leeuwenhoek, 81(1-4), 537-547. 
Spadaro, D., M. Gullino, 2005. Improving the efficacy of biocontrol agents against soilborne pathogens. Crop Prot., 24(7), 601-613.

Satish, S., M. Raghavendra, K. Raveesha, 2009. Antifungal potential of some plant extracts against Fusarium sp. Arch. Phytopathology Plant Protect., 42(7), 618-625.

Soxhlet, F., 1879. Die gewichtsanalytische Bestimmung des Milchfettes. Polytechnisches J., 232, 461. 